\title{
The genome sequence of the grey top shell, Steromphala
}

\section{cineraria (Linnaeus, 1758) [version 1; peer review: awaiting}

\section{peer review]}

\section{Patrick Adkins¹, Robert Mrowicki (iD1,2, Joanna Harley1, Nova Mieszkowska(i1, Marine Biological Association Genome Acquisition Lab, Darwin Tree of Life Barcoding collective, Wellcome Sanger Institute Tree of Life programme, Wellcome Sanger Institute Scientific Operations: DNA Pipelines collective, Tree of Life Core Informatics collective, Darwin Tree of Life Consortium}

${ }^{1}$ Marine Biological Association, Plymouth, Devon, UK

${ }^{2}$ Natural History Museum, London, UK

V1 First published: 08 Feb 2022, 7:44

https://doi.org/10.12688/wellcomeopenres.17677.1

Latest published: 24 Mar 2022, 7:44

https://doi.org/10.12688/wellcomeopenres.17677.2

\section{Abstract}

We present a genome assembly from an individual Steromphala cineraria (the grey topshell; Mollusca; Gastropoda; Trochida; Trochidae). The genome sequence is 1,270 megabases in span. The majority of the assembly (99.23\%) is scaffolded into 18 chromosomal pseudomolecules.

\section{Keywords}

Steromphala cineraria, grey topshell, genome sequence, chromosomal, Mollusca

This article is included in the Tree of Life gateway.

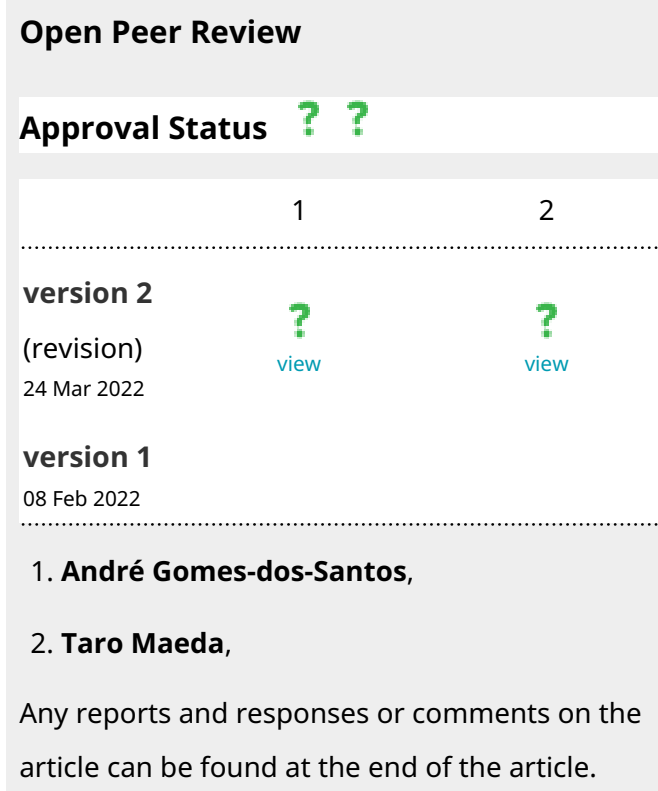


Corresponding author: Darwin Tree of Life Consortium (mark.blaxter@sanger.ac.uk)

Author roles: Adkins P: Investigation, Resources, Writing - Original Draft Preparation, Writing - Review \& Editing; Mrowicki R: Investigation, Resources; Harley J: Investigation, Resources; Mieszkowska N: Supervision, Writing - Original Draft Preparation, Writing Review \& Editing;

Competing interests: No competing interests were disclosed.

Grant information: This work was supported by Wellcome through core funding to the Wellcome Sanger Institute (206194) and the Darwin Tree of Life Discretionary Award (218328).

The funders had no role in study design, data collection and analysis, decision to publish, or preparation of the manuscript.

Copyright: (c) 2022 Adkins P et al. This is an open access article distributed under the terms of the Creative Commons Attribution License, which permits unrestricted use, distribution, and reproduction in any medium, provided the original work is properly cited.

How to cite this article: Adkins P, Mrowicki R, Harley J et al. The genome sequence of the grey top shell, Steromphala cineraria (Linnaeus, 1758) [version 1; peer review: awaiting peer review] Wellcome Open Research 2022, 7:44

https://doi.org/10.12688/wellcomeopenres.17677.1

First published: 08 Feb 2022, 7:44 https://doi.org/10.12688/wellcomeopenres.17677.1 


\section{Species taxonomy}

Eukaryota; Metazoa; Spiralia; Lophotrochozoa; Mollusca; Gastropoda; Vetigastropoda; Trochida; Trochoidea; Trochidae; Cantharidinae; Steromphala; Steromphala cineraria (Linnaeus, 1758) (NCBI:txid216125).

\section{Background}

Steromphala cineraria (Linnaeus, 1758), commonly called the grey topshell, is a gastropod common to rocky shores in the UK. It typically occurs among boulders and cobbles on the lowshore and sub-tidally, where it grazes among Fucus and Laminaria species. Intertidally, it is most common on the lower shore, but can also be found in pools higher on the shore. Sub-tidally it extends to depths of $130 \mathrm{~m}$, although it is most common in the kelp forests between $30 \mathrm{~m}$ and low water spring tide (Fretter \& Graham, 1976). Its geographical distribution ranges from southern Portugal and north to the White Sea in northern Russia, becoming rarer at its range edges as thermal limits are approached (Nekhaev, 2013).

An important grazing species, $S$. cineraria is distinguished from other species of trochids by its bluntly conical shell and grey/yellowish finely striped patterning on the shell. In smaller shells, the umbilicus is large, becoming smaller and elliptical with age and in large shells sometimes becoming overgrown by the columellar lip (Fretter \& Graham, 1976).

As $S$. cineraria is found across a large range of latitudes, it is exposed to a wide range of thermal environments in temperature, both due to time of year and geographical distribution. It is important to understand how populations may change in response to climate change, especially in its southern and northern range limits, and the knock-on effects this may have on macroalgae due to changes in grazing populations (Mieszkowska et al., 2007). A high quality genome sequence for this species will allow future studies to understand more about the mechanisms driving the observed response of this species to a changing climate.

\section{Genome sequence report}

The genome was sequenced from a single $S$. cineraria (Figure 1) collected from Mount Batten, Devon, UK (latitude 50.36084, longitude -4.12833). A total of 42-fold coverage in Pacific Biosciences single-molecule long reads and 35-fold coverage in 10X Genomics read clouds were generated. Primary assembly contigs were scaffolded with chromosome conformation Hi-C data. Manual assembly curation corrected $408 \mathrm{missing} / \mathrm{misjoins}$ and removed 70 haplotypic duplications, reducing the assembly size by $2.51 \%$ and the scaffold number by $53.835 \%$, and increasing the scaffold N50 by $123.17 \%$.

The final assembly has a total length of $1,270 \mathrm{Mb}$ in 283 sequence scaffolds with a scaffold N50 of $70.7 \mathrm{Mb}$ (Table 1). Of the assembly sequence, $99.23 \%$ was assigned to 18 chromosomal-level scaffolds (numbered by sequence length) (Figure 2Figure 5; Table 2). Large inversions between sister chromatids can be seen on chromosome 5 at 29.7-60.7 Mb and chromosome 11 at $17.7-39.7 \mathrm{Mb}$. Possible inversions are also seen on chromosome 11 at $\mathrm{Mb} 3.4-39.4$ and 18-66 Mb. The assembly has a BUSCO v5.1.2 (Manni et al., 2021) completeness of $85.4 \%$ (single $84.6 \%$, duplicated $0.8 \%$ ) using the mollusca_odb 10 reference set $(n=5295)$. However, we believe that this relatively low BUSCO score is a result of limitations with the current mollusca_odb10 geneset. Using the metazoa_odb10 reference set

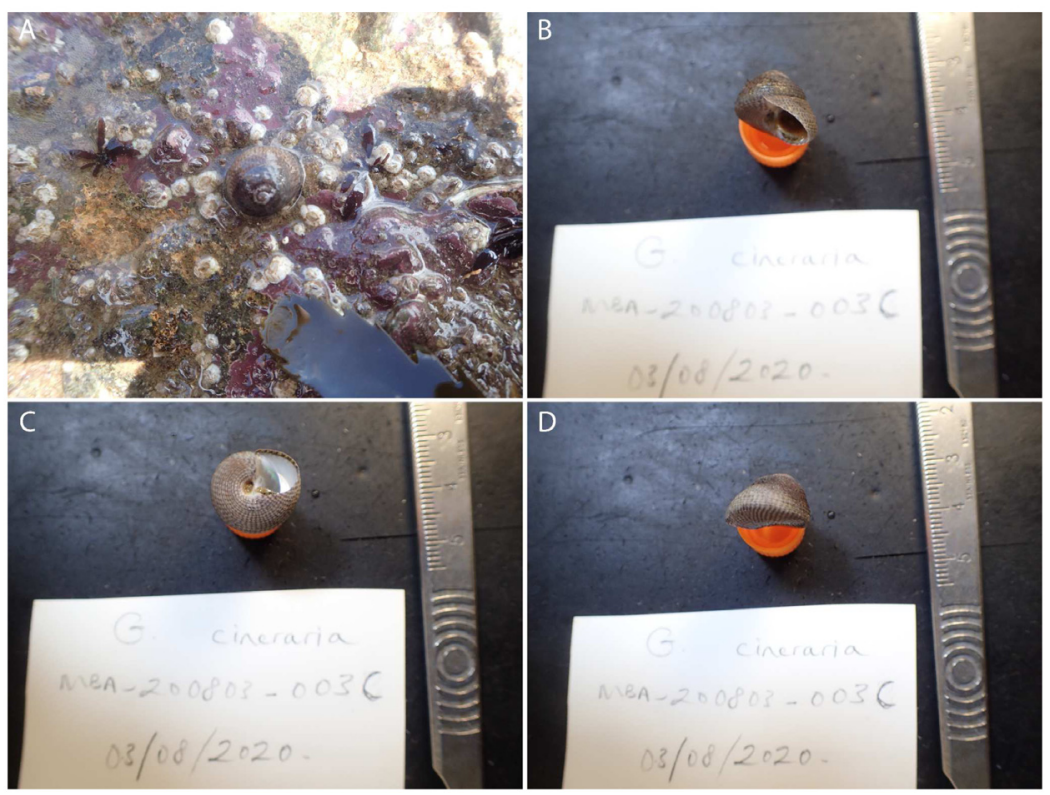

Figure 1. Image of the $\mathbf{x g S t e C i n e 2}$ specimen. (A) Image taken of the specimen prior to collection. (B-D) Image of the shell of the specimen following preservation and processing. 


\begin{tabular}{|c|c|}
\hline \multicolumn{2}{|l|}{ Project accession data } \\
\hline Assembly identifier & xgSteCine2.1 \\
\hline Species & Steromphala cineraria \\
\hline Specimen & xgSteCine2 \\
\hline NCBI taxonomy ID & NCBI:txid216125 \\
\hline BioProject & PRJEB45667 \\
\hline BioSample ID & SAMEA7536348 \\
\hline Isolate information & Muscle \\
\hline \multicolumn{2}{|l|}{ Raw data accessions } \\
\hline PacificBiosciences SEQUEL II & ERR6939216, ERR6939217 \\
\hline 10X Genomics Illumina & ERR6363284-ERR6363287 \\
\hline Hi-C Illumina & ERR6363289 \\
\hline PolyA RNA-Seq Illumina & ERR6688409 \\
\hline \multicolumn{2}{|l|}{ Genome assembly } \\
\hline Assembly accession & GCA_916613615.1 \\
\hline Accession of alternate haplotype & GCA_916613985.1 \\
\hline Span (Mb) & 1,270 \\
\hline Number of contigs & 842 \\
\hline Contig N50 length (Mb) & 6.2 \\
\hline Number of scaffolds & 283 \\
\hline Scaffold N50 length (Mb) & 70.7 \\
\hline Longest scaffold (Mb) & 98.8 \\
\hline BUSCO* genome score & $\begin{array}{l}\text { C:85.4\%[S:84.6\%,D:0.8\%], } \\
\text { F:4.9\%,M:9.7\%,n:5295 }\end{array}$ \\
\hline
\end{tabular}

*BUSCO scores based on the hymenoptera_odb10 BUSCO set using v5.1.2. $C=$ complete $[S=$ single copy, $D=$ duplicated], $F=$ fragmented, $\mathrm{M}=$ missing, $\mathrm{n}=$ number of orthologues in comparison. A full set of BUSCO scores is available at https://blobtoolkit.genomehubs.org/view/ Steromphala\%20cineraria/dataset/CAKAJN01/busco.

( $n=954$ ), the assembly has a completeness of $97.6 \%$ (single $97.0 \%$, duplicated $0.6 \%$ ), which we believe is evidence of high completeness. While not fully phased, the assembly deposited is of one haplotype. Contigs corresponding to the second haplotype have also been deposited.

\section{Methods}

Sample acquisition and nucleic acid extraction

A single $S$. cineraria specimen (xgSteCine2) was collected from Mount Batten, Devon, UK (latitude 50.36084, longitude -4.12833) by Rob Mrowicki (Natural History Museum), Patrick Adkins and Joanna Harley (both Marine Biological
Association), by hand. The samples were identified by the same individual and snap-frozen in liquid nitrogen.

DNA was extracted at the Tree of Life laboratory, Wellcome Sanger Institute. The xgSteCine2 sample was weighed and dissected on dry ice with tissue set aside for Hi-C and RNA sequencing. Muscle tissue was cryogenically disrupted to a fine powder using a Covaris cryoPREP Automated Dry Pulveriser, receiving multiple impacts. Fragment size analysis of $0.01-0.5 \mathrm{ng}$ of DNA was then performed using an Agilent FemtoPulse. High molecular weight (HMW) DNA was extracted using the Qiagen MagAttract HMW DNA extraction kit. Low molecular weight 


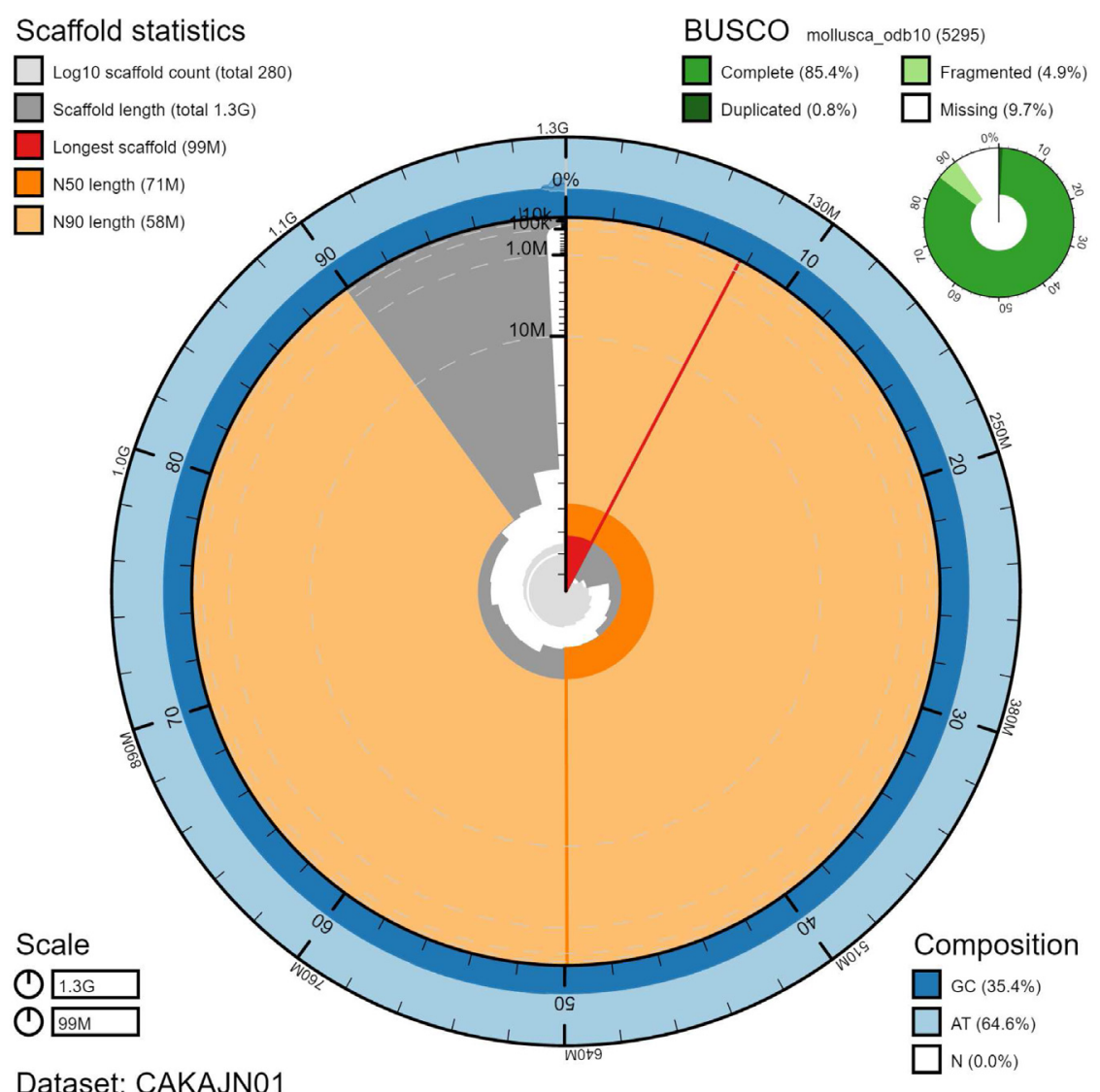

Figure 2. Genome assembly of Steromphala cineraria, xgSteCine2.1: metrics. The BlobToolKit Snailplot shows N50 metrics and BUSCO gene completeness. The main plot is divided into 1,000 size-ordered bins around the circumference with each bin representing $0.1 \%$ of the 1,270,504,078 bp assembly. The distribution of scaffold lengths is shown in dark grey with the plot radius scaled to the longest scaffold present in the assembly $(98,775,408 \mathrm{bp}$, shown in red). Orange and pale-orange arcs show the N50 and N90 scaffold lengths $(70,748,747$ and $57,769,559$ bp), respectively. The pale grey spiral shows the cumulative scaffold count on a log scale with white scale lines showing successive orders of magnitude. The blue and pale-blue area around the outside of the plot shows the distribution of GC, AT and $\mathrm{N}$ percentages in the same bins as the inner plot. A summary of complete, fragmented, duplicated and missing BUSCO genes in the mollusca_odb10 set is shown in the top right. An interactive version of this figure is available at https://blobtoolkit.genomehubs.org/view/ Steromphala\%20cineraria/dataset/CAKAJN01/snail.

DNA was removed from a 200-ng aliquot of extracted DNA using $0.8 \mathrm{X}$ AMpure XP purification kit prior to $10 \mathrm{X}$ Chromium sequencing; a minimum of $50 \mathrm{ng}$ DNA was submitted for $10 \mathrm{X}$ sequencing. HMW DNA was sheared into an average fragment size between $12-20 \mathrm{~kb}$ in a Megaruptor 3 system with speed setting 30. Sheared DNA was purified by solid-phase reversible immobilisation using AMPure PB beads with a $1.8 \mathrm{X}$ ratio of beads to sample to remove the shorter fragments and concentrate the DNA sample. The concentration of the sheared and purified DNA was assessed using a Nanodrop spectrophotometer and Qubit Fluorometer and Qubit dsDNA High Sensitivity Assay kit. Fragment size distribution was evaluated by running the sample on the FemtoPulse system.

RNA was extracted from muscle tissue in the Tree of Life Laboratory at the WSI using TRIzol, according to the manufacturer's instructions. RNA was then eluted in $50 \mu \mathrm{l}$ RNAse-free water and its concentration RNA assessed using a
Nanodrop spectrophotometer and Qubit Fluorometer using the Qubit RNA Broad-Range (BR) Assay kit. Analysis of the integrity of the RNA was done using Agilent RNA 6000 Pico Kit and Eukaryotic Total RNA assay.

\section{Sequencing}

Pacific Biosciences HiFi circular consensus and 10X Genomics read cloud sequencing libraries were constructed according to the manufacturers' instructions. Sequencing was performed by the Scientific Operations core at the Wellcome Sanger Institute on Pacific Biosciences SEQUEL II and Illumina NovaSeq 6000 instruments. Hi-C data were generated from additional muscle tissue of $\mathrm{xgSteCine} 2$ using the Arima v2.0 kit and sequenced on an Illumina NovaSeq 6000 instrument.

\section{Genome assembly}

Assembly was carried out with Hifiasm (Cheng et al., 2021). Haplotypic duplication was identified and removed with 


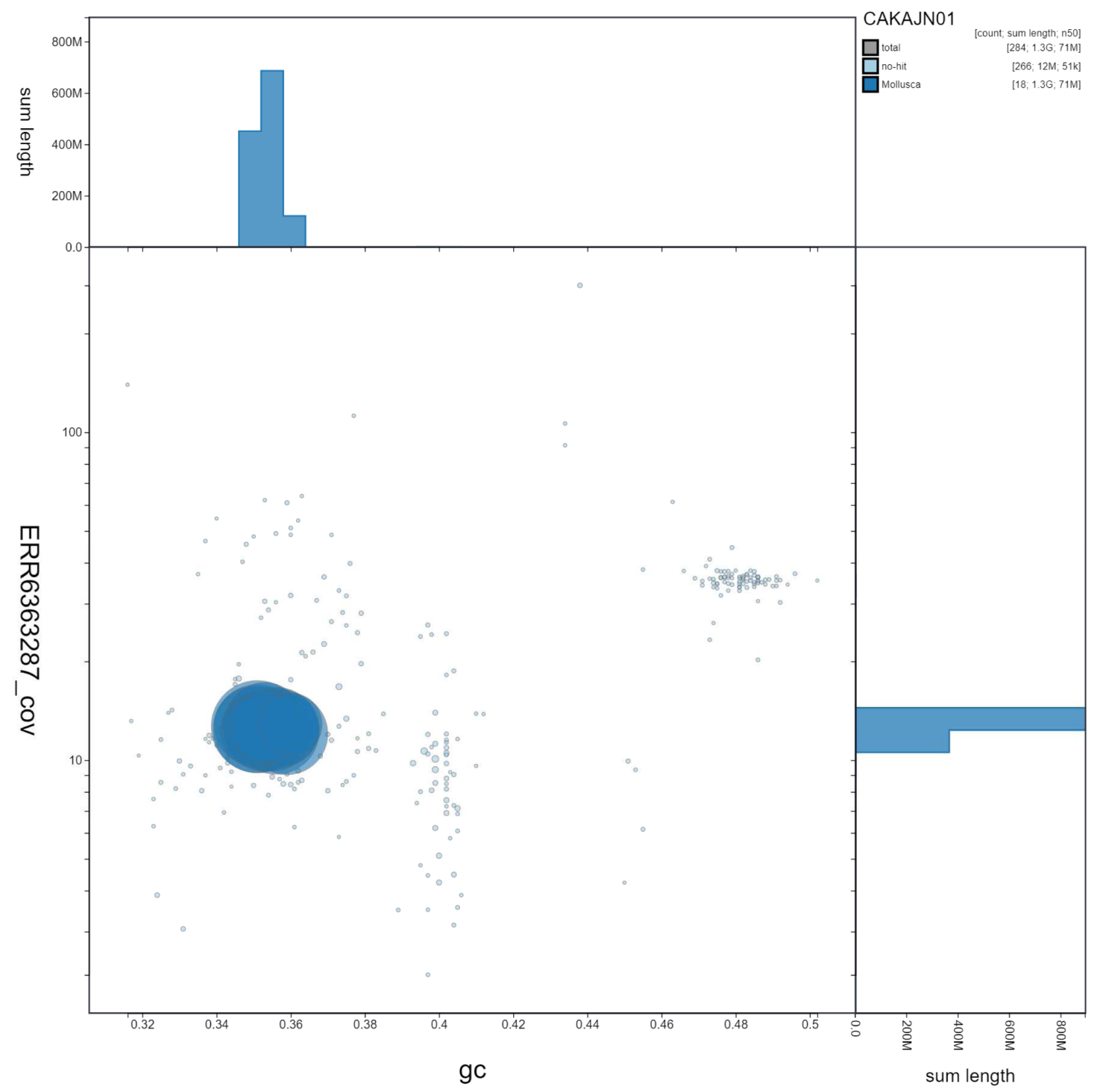

Figure 3. Genome assembly of Steromphala cineraria, xgSteCine2.1. GC coverage. BlobToolKit GC-coverage plot. Scaffolds are coloured by phylum. Circles are sized in proportion to scaffold length. Histograms show the distribution of scaffold length sum along each axis. An interactive version of this figure is available at https://blobtoolkit.genomehubs.org/view/Steromphala\%20cineraria/dataset/ CAKAJN01/blob.

purge_dups (Guan et al., 2020). One round of polishing was performed by aligning $10 \mathrm{X}$ Genomics read data to the assembly with longranger align, calling variants with freebayes (Garrison \& Marth, 2012). The assembly was then scaffolded with Hi-C data (Rao et al., 2014) using SALSA (Ghurye et al., 2019). The mitochondrial genome was assembled with MitoHiFi (Uliano-Silva et al., 2021), which performed annotation using MitoFinder (Allio et al., 2020). The assembly was checked for contamination and corrected using the gEVAL system (Chow et al., 2016) as described previously (Howe et al., 2021). Manual curation (Howe et al., 2021) was performed using gEVAL, HiGlass (Kerpedjiev et al., 2018) and Pretext. The genome was analysed within the BlobToolKit environment (Challis et al., 2020). Table 3 contains a list of all software tool versions used, where appropriate.

\section{Ethics/compliance issues}

The materials that have contributed to this genome note have been supplied by a Darwin Tree of Life Partner. The submission of materials by a Darwin Tree of Life Partner is subject to the Darwin Tree of Life Project Sampling Code of Practice. By agreeing with and signing up to the Sampling Code of Practice, the Darwin Tree of Life Partner agrees they will meet the legal and ethical requirements and standards set out within this document in respect of all samples acquired for, and supplied to, the Darwin Tree of Life Project. Each transfer of 


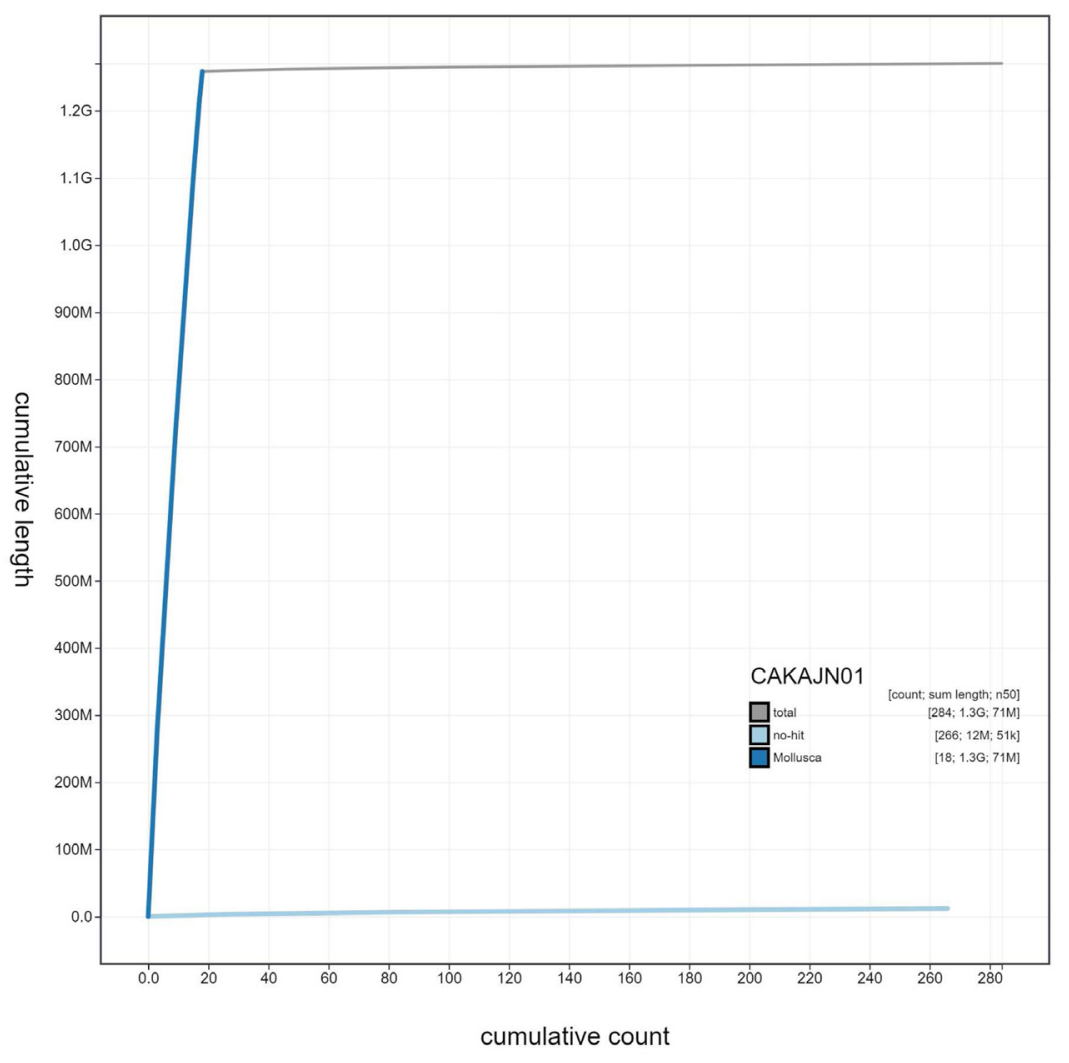

Figure 4. Genome assembly of Steromphala cineraria, xgSteCine2.1: cumulative sequence. BlobToolKit cumulative sequence plot. The grey line shows cumulative length for all scaffolds. Coloured lines show cumulative lengths of scaffolds assigned to each phylum using the buscogenes taxrule. An interactive version of this figure is available at https://blobtoolkit.genomehubs.org/view/Steromphala\%20cineraria/ dataset/CAKAJN01/cumulative.

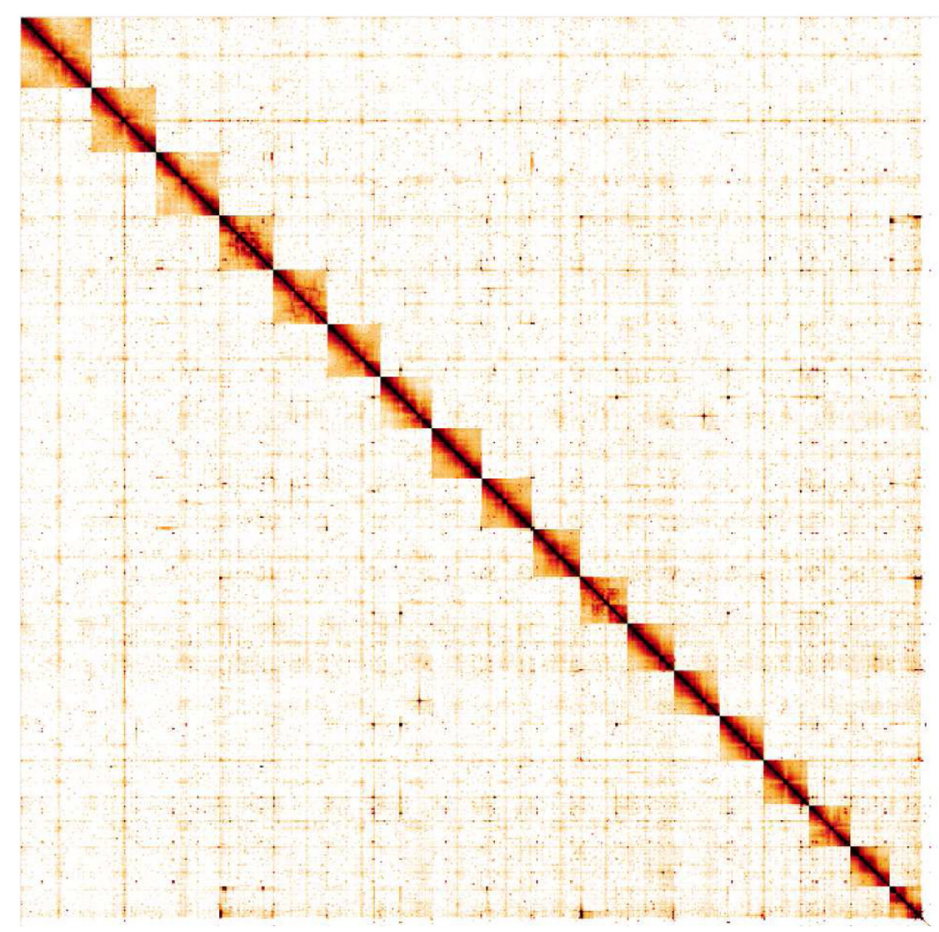

Figure 5. Genome assembly of Steromphala cineraria, xgSteCine2.1: Hi-C contact map. Hi-C contact map of the xgSteCine2.1 assembly, visualised in HiGlass. Chromosomes are shown in size order from left to right and top to bottom. 


\begin{tabular}{|c|c|c|c|}
\hline $\begin{array}{l}\text { Table 2. Chromosomal pseudomolecules in the } \\
\text { genome assembly of Steromphala cineraria } \\
\text { xgSteCine2.1. }\end{array}$ \\
\hline INSDC accession & Chromosome & Size (Mb) & GC\% \\
\hline OU744720.1 & 1 & 98.78 & 35.1 \\
\hline OU744721.1 & 2 & 90.22 & 35.1 \\
\hline OU744722.1 & 3 & 87.49 & 35.6 \\
\hline OU744723.1 & 4 & 76.76 & 35.9 \\
\hline OU744724.1 & 5 & 75.56 & 35.5 \\
\hline OU744725.1 & 6 & 74.15 & 35.3 \\
\hline OU744727.1 & 7 & 70.75 & 35.0 \\
\hline OU744726.1 & 8 & 71.35 & 35.3 \\
\hline OU744728.1 & 9 & 70.17 & 35.7 \\
\hline OU744729.1 & 10 & 66.46 & 35.2 \\
\hline OU744730.1 & 11 & 66.15 & 35.5 \\
\hline OU744731.1 & 12 & 65.37 & 35.3 \\
\hline OU744732.1 & 13 & 62.64 & 35.1 \\
\hline OU744733.1 & 14 & 62.10 & 35.2 \\
\hline OU744734.1 & 15 & 61.92 & 35.4 \\
\hline OU744735.1 & 16 & 57.77 & 35.8 \\
\hline OU744736.1 & 17 & 56.52 & 35.3 \\
\hline OU744737.1 & 18 & 44.44 & 36.0 \\
\hline OU744738.1 & MT & 31.6 \\
\hline- & 0.02 & 39.8 \\
\hline
\end{tabular}

\begin{tabular}{|c|c|c|}
\hline Software tool & Version & Source \\
\hline Hifiasm & 0.15 & Cheng et al., 2021 \\
\hline purge_dups & 1.2 .5 & Guan et al., 2020 \\
\hline SALSA2 & 3.0 & Ghurye et al., 2019 \\
\hline longranger align & 2.2 .2 & $\begin{array}{l}\text { https://support.10xgenomics.com/genome-exome/ } \\
\text { software/pipelines/latest/advanced/other-pipelines }\end{array}$ \\
\hline freebayes & v1.3.1 & Garrison \& Marth, 2012 \\
\hline MitoHiFi & 2 & https://github.com/marcelauliano/MitoHiFi \\
\hline gEVAL & N/A & Chow et al., 2016 \\
\hline HiGlass & 1.11 .6 & Kerpedjiev et al., 2018 \\
\hline PretextView & $0.2 . x$ & https://github.com/wtsi-hpag/PretextView \\
\hline BlobToolKit & 2.6 .4 & Challis et al., 2020 \\
\hline
\end{tabular}


samples is further undertaken according to a Research Collaboration Agreement or Material Transfer Agreement entered into by the Darwin Tree of Life Partner, Genome Research Limited (operating as the Wellcome Sanger Institute), and in some circumstances other Darwin Tree of Life collaborators.

\section{Data availability}

European Nucleotide Archive: Steromphala cineraria (grey top shell). Accession number PRJEB45667; https://identifiers.org/ena. embl/PRJEB45667.

The genome sequence is released openly for reuse. The $S$. cineraria genome sequencing initiative is part of the Darwin Tree of Life (DToL) project. All raw sequence data and the assembly have been deposited in INSDC databases. The genome will be annotated with the RNA-Seq data and presented through the Ensembl pipeline at the European Bioinformatics Institute. Raw data and assembly accession identifiers are reported in Table 1 .

\section{Author information}

Members of the Marine Biological Association Genome Acquisition Lab are listed here: https://doi.org/10.5281/ zenodo.5913830.

Members of the Darwin Tree of Life Barcoding collective are listed here: https://doi.org/10.5281/zenodo.5744972.

Members of the Wellcome Sanger Institute Tree of Life programme are listed here: https://doi.org/10.5281/zenodo.5744840.

Members of Wellcome Sanger Institute Scientific Operations: DNA Pipelines collective are listed here: https://doi.org/10.5281/ zenodo. 5746904

Members of the Tree of Life Core Informatics collective are listed here: https://doi.org/10.5281/zenodo.5743293.

Members of the Darwin Tree of Life Consortium are listed here: https://doi.org/10.5281/zenodo.5638618.
Allio R, Schomaker-Bastos A, Romiguier J, et al.: MitoFinder: Efficient Automated Large-Scale Extraction of Mitogenomic Data in Target Enrichment Phylogenomics. Mol Ecol Resour. 2020; 20(4): 892-905. PubMed Abstract | Publisher Full Text | Free Full Text

Challis R, Richards E, Rajan J, et al.: BlobToolKit - Interactive Quality Assessment of Genome Assemblies. G3 (Bethesda). 2020; 10(4): 1361-74. PubMed Abstract | Publisher Full Text | Free Full Text

Cheng $\mathrm{H}$, Concepcion GT, Feng $\mathrm{X}$, et al.: Haplotype-Resolved de Novo Assembly Using Phased Assembly Graphs with Hifiasm. Nat Methods. 2021; 18(2): 170-75.

PubMed Abstract | Publisher Full Text | Free Full Text

Chow W, Brugger K, Caccamo M, et al.: gEVAL - a Web-Based Browser for Evaluating Genome Assemblies. Bioinformatics. 2016; 32(16): 2508-10. PubMed Abstract | Publisher Full Text | Free Full Text

Fretter V, Graham A: The Prosobranch Molluscs of Britain and Denmark:

Part 2. Trochacea. The Journal of Molluscan Studies. 1976; 42.

Reference Source

Garrison E, Marth G: Haplotype-Based Variant Detection from Short-Read Sequencing. arXiv: 1207.3907, 2012

Reference Source

Ghurye J, Rhie A, Walenz BP, et al.: Integrating Hi-C Links with Assembly Graphs for Chromosome-Scale Assembly. PLoS Comput Biol. 2019; 15(8): e1007273. PubMed Abstract | Publisher Full Text | Free Full Text

Guan D, McCarthy SA, Wood J, et al.: Identifying and Removing Haplotypic Duplication in Primary Genome Assemblies. Bioinformatics. 2020; 36(9): 2896-98.

PubMed Abstract | Publisher Full Text | Free Full Text
Howe K, Chow W, Collins J, et al:: Significantly Improving the Quality of Genome Assemblies through Curation. GigaScience. 2021; 10(1): giaa153. PubMed Abstract | Publisher Full Text | Free Full Text

Kerpedjiev P, Abdennur N, Lekschas F, et al.: HiGlass: Web-Based Visual Exploration and Analysis of Genome Interaction Maps. Genome Biol. 2018; 19(1): 125.

PubMed Abstract | Publisher Full Text | Free Full Text

Manni M, Berkeley MR, Seppey M, et al.: BUSCO Update: Novel and

Streamlined Workflows along with Broader and Deeper Phylogenetic Coverage for Scoring of Eukaryotic, Prokaryotic, and Viral Genomes. Mol Biol Evol. 2021; 38(10): 4647-54.

PubMed Abstract | Publisher Full Text | Free Full Text

Mieszkowska N, Kendall MA, Hawkins SJ, et al.: Changes in the Range of Some Common Rocky Shore Species In Britain - A Response To Climate Change? In. Developments in Hydrobiology. 2007; 241-51.

Publisher Full Text

Nekhaev IO: Distributional Notes on Gibbula Cineraria (Linnaeus, 1758), Pseudosetia Turgida (Jeffreys, 1870) and Haliella Stenostoma (Jeffreys, 1858) in Russian Part of the Barents Sea (Gastropoda). Ruthenica. 2013; 23(1). Reference Source

Rao SSP, Huntley MH, Durand NC, et al:: A 3D Map of the Human Genome at Kilobase Resolution Reveals Principles of Chromatin Looping. Cell. 2014; 159(7): 1665-80.

PubMed Abstract | Publisher Full Text | Free Full Text

Uliano-Silva M, Nunes JGF, Krasheninnikova K, et al:: marcelauliano/MitoHiFi: mitohifi_v2.0.2021.

Publisher Full Text 